\title{
Estudios sobre la corrección de la disgrafía caligráfica en escolares con discapacidad intelectual
}

\section{Studies on the correction of calligraphic dysgraphia in schoolchildren with intellectual disabilities}

\author{
Yisel Santana del Sol \\ Centro Universitario Municipal Palmira. Universidad de Cienfuegos, Cuba \\ ORCID: https://orcid.org/0000-0002-4416-4109 \\ Karel LLópiz Guerra \\ Universidad Central "Marta Abreu" de las Villas, Cuba. \\ ORCID: https://orcid.org/0000-0002-1500-8000 \\ Marilyn Oderay Sugasty Medina \\ Instituto de Panameño de Habilitación Especial, Panamá. \\ Aracelli Gonzales-Sánchez \\ Universidad César Vallejo, Perú \\ ORCID: https://orcid.org/0000-0003-0028-9177 \\ José Mercedes Valqui Oxolon \\ Universidad Cesar Vallejo, Perú. \\ ORCID: https://orcid.org/0000-0003-0849-9080
}

*Correspondence

Email: ysantanas@ucf.edu.cu
Cite as: 


\section{Resumen}

En este artículo se realiza un estudio teórico relacionado con la corrección de la disgrafía caligráfica, así como las características de los escolares con discapacidad intelectual con este tipo de trastorno. Se revisaron teorías y concepciones existentes acerca de la corrección de la digrafía caligráfica, identificando las fallas o síntomas que presentan estos escolares con discapacidad intelectual. Para una educación con calidad, resulta conveniente señalar, la importancia que reviste la atención educativa integral al escolar con necesidad educativa especial en la comunicación escrita, de ahí la necesidad de prepararlo en la corrección de los trastornos de escritura, que necesitan de métodos y alternativas especializadas, en función de su desarrollo.

Palabras claves: Corrección, Disgrafía Caligráfica, Discapacidad Intelectual.

\section{Sunnary}

This article analyzes theoretical aspects related to the correction of calligraphic dysgraphia, as well as the characteristics of schoolchildren with intellectual disabilities with this type of disorder. Existing theories and conceptions about correcting calligraphic dysgraphia were reviewed, identifying the flaws or symptoms presented by these schoolchildren with intellectual disabilities. For a quality education, it is convenient to point out, the importance of comprehensive educational care for students with special educational needs in written communication, hence the need to prepare them to correct writing disorders, which require methods and alternatives specialized, depending on their development. The criterion of practice, through the use of actions in the school and the criterion of experts, allows to guarantee its possibility, aptitude and value to originate the changes that the transformations require at the level and develop the integral education of the new man.

Keywords: Correction, Calligraphic Dysgraphia, Intellectual Disability.

\section{Introducción}

La Agenda 2030 proporciona un nuevo empuje a las acciones de la UNESCO a nivel nacional, regional y global, concede prioridad a la contribución de la educación inclusiva y de calidad en todos los niveles y a la importancia de las oportunidades del aprendizaje permanente para todos, Objetivo de Desarrollo Sostenible (ODS 4). Defiende una visión de sociedades justas, pacíficas, equitativas e inclusivas, reconociendo el valor del conocimiento, el patrimonio y la diversidad. La UNESCO (2020) aborda la ejecución de la Agenda 2030 de forma integrada a través de todas sus áreas de competencia (educación, ciencias naturales, sociales y humanas, cultura, comunicación e información), reconoce la importancia de abordar las crecientes desigualdades mediante su trabajo en promover la educación inclusiva y de calidad para todos; acortando las brechas en ciencias y conocimiento entre y dentro de los países; cerrando la divisoria digital; y desarrollando políticas públicas integradoras que promocionen la inclusión social y el diálogo intercultural; ha sido decisiva en la formulación de esta agenda, plasmada en el Objetivo autónomo de Desarrollo Sostenible 4 (ODS 4).

Muchas de las políticas educacionales se sustentan en el reconocimiento de la escuela como la institución a la que ha sido conferida la alta misión de guiar la formación de las jóvenes generaciones, para lo cual dirige el proceso docente educativo y coordina el resto de las influencias educativas, por eso se concibe que se preste especial atención a niños con necesidades educativas especiales.

La escuela, prepara para la vida a niños y jóvenes en correspondencia con las posibilidades creadas por el medio social y con vistas a dar respuesta a las necesidades que supone su desarrollo, de ahí que deba responder a las demandas que emergen a escala 
internacional y al mismo tiempo adecuarse a las realidades de nuestro contexto nacional, con énfasis en los problemas propios de los territorios, las familias y todas las personas participantes en el proceso educativo.

Por ello se profundiza en el principal factor que hace posible el desarrollo de los educandos considerándose así al aprendizaje como un proceso que ocurre a lo largo de la vida de los seres humanos en cada una de las situaciones que colocan al hombre en permanente interacción con la cultura. Su análisis exige la delimitación de dos dimensiones: individual y social, pues la apropiación de los elementos de la cultura es personal y se da en interacción con otros en un momento del desarrollo histórico social.

Las personas con discapacidad intelectual forman un grupo que se distingue por su diversidad, comparten un conjunto de regularidades que están determinadas en su generalidad por la interacción entre los aspectos de orden biológico, psicológico y social, aunque cada ser humano es único e irrepetible.

La atención educativa a los escolares con discapacidad intelectual constituye una prioridad que deben asumir los diferentes profesionales implicados en su educación, así como los miembros de su familia y otros agentes educativos, por lo cual se presta especial atención a escolares que presenten este trastorno y principalmente en el desarrollo del lenguaje escrito, el cual tiene la misma estructura eslabonada propia del lenguaje oral y por tanto presenta trastornos que pueden ubicarse de los niveles motivacionales e ideáticos hasta los propios de la programación articulatoria.

Esta forma de lenguaje comprende los procesos de escritura y los de la lectura, por lo que como objetivo de este trabajo se realiza un estudio acerca de los trastornos del lenguaje escrito, y las vías de corrección de la disgrafía caligráfica en diferentes latitudes, identificando las fallas o síntomas que presentan estos escolares con discapacidad intelectual.

\section{Argumentación}

Los trastornos de la escritura pueden presentarse a cualquier edad. No solo son una consecuencia de deficiencias en los mecanismos que facilitan su desarrollo; si las premisas anatomofisiológicas, psicológicas y sociales no están dadas, esta forma de lenguaje escrito no se desarrolla o se forma insuficientemente. También se pueden presentar estados patológicos como resultado de deterioros en los mecanismos establecidos.

Debido a lo anteriormente mencionado se realiza un estudio a profundidad de los aportes que se han realizado desde la proyectiva de la atención preventiva en estos casos, el tema en cuestión absorbe el interés de pedagogos y psicólogos por lo que constituye una línea priorizada de las investigaciones actuales. Muestra de ello son los trabajos de varios autores internacionales citados por Pérez, G.F. (2013) tales como: Vygotsky (1989), Verdugo (1995) Acudovich (2004), los cuales han realizado un trabajo profundo y minucioso sobre el tema. Loaiza (2015), expresa que: Para una correcta escritura es necesario el desarrollo normal de habilidades y destrezas de motricidad. La autora afirma que esto, muchas veces se ve seccionado cuando los estudiantes tienen problemas de disgrafía, misma que se presenta ante la dificultad de coordinar los músculos de la mano y el brazo impidiendo de esta forma dominar los instrumentos de escritura como el denominado lápiz.

En Cuba se han ido destacando sobre este tema se encuentran autores como Gayle (2004), Guerra (2005), López (2010), Orozco (2012) y Borges (2012). Estos dos últimos han indagado sobre la Educación Inclusiva y abordan en su contenido acerca de la atención a los escolares con discapacidad intelectual. Otras investigaciones desarrolladas sobre esta línea se desarrollan en el perfil de Rodríguez (2008) y Chávez (2009), los cuales reconocen las 
capacidades y habilidades que pueden presentar los escolares con discapacidad intelectual para enfrentar el currículo con el nuevo perfeccionamiento y los retos de la vida cotidiana.

Diversos han sido los autores que han abordado el tema relacionado con la disgrafía a nivel internacional como Torres (1980), Herrera (1988) y Morenza (1996) y en el ámbito nacional: Padrón (2002), Martínez y Col (2003), Cobas (2013) y Fernández (2013), los cuales abordan las causas, las características y los tipos de disgrafia y proponen las etapas para el tratamiento de la disgrafia. Abad (2008), elaboró una propuesta de actividades para corregir la disgrafia sensorial y óptico-espacial en los escolares de segundo grado. R.L. Miranda (2008) con resultados muy favorables, desarrolló una propuesta de actividades encaminadas a preparar a los niños bajos en el diagnóstico de preescolar para la adquisición de la lecto-escritura en 1er grado.

La lengua escrita es la forma y corporeidad del lenguaje representado por las combinaciones de signos gráficos que hacen posible la formulación de lo contenido en el pensamiento y se convierte en algo consciente, que puede valorarse y considerarse un sistema creado por el hombre para representar objetivamente o transcribir la realidad oral en que la lengua coexiste, comprende los procesos de la lectura y la escritura; como canal de comunicación, incluye los procesos de codificación y decodificación de formas gráficas.

Estos constituyen menesteres esenciales en la formación cultural del individuo, que hace posible avanzar y elevarse en los grados de solidez de los conocimientos y del enriquecimiento espiritual y humano.

La disgrafia por su parte es un trastorno de tipo funcional que afecta a la calidad de la escritura del sujeto, en el trazado o la grafía. Se llama disgráfico al que confunde, omite, une o invierte sílabas o letras de forma incorrecta. Se considera como disgráfico al alumno que comete dos o más tipos de incorrecciones, al que tiene las aptitudes mentales y sensoriales normales y ha sido escolarizado. El número de casos es mayor en los niños, con un $60 \%$ más que en las niñas. Anderson (2014).

En el cumplimiento de los objetivos trazados en el contexto institucional el maestro debe orientarse adecuadamente para intensificar la individualización y diferenciación sin barrera con aquellos estudiantes que comienzan desde los primeros grados a manifestar necesidades educativas que atentan con el desarrollo exitoso de su aprendizaje.

Para evitar el agravamiento de estas dificultades que se presentan en el contexto escolar se hace necesario profundizar en algunos aspectos importantes que facilitarían la labor pedagógica. Para ello es necesario precisar qué se entiende por corrección y compensación, tomado de Medina, Rivero y Morales (2013):

Corrección: Eliminar, acercar a las características de la conducta normal, devolver a la norma las funciones y conducta deseada. Es un elemento del proceso de rehabilitación. Corregir significa modificar, eliminar las deficiencias conductuales de los alumnos, mejorar sus procesos psíquicos.

Compensación: Complejo y variado proceso de reestructuración de las funciones del organismo en caso de trastornos o pérdida de cualquiera de ellas, a causa de enfermedades padecidas o de lesiones traumáticas.

En la asignatura Lengua Española el alumno adquiere el instrumento de la escritura y se establece la adecuada correspondencia entre la lengua oral y escrita de este modo se establecen desde el primer momento vínculos entre la operación decodificadora de leer y la operación codificadora de escribir y la relación dialéctica que existe entre ambos.

Los propósitos primordiales de la enseñanza de la escritura son capacitar a cada escolar para escribir de manera legible y con una velocidad razonable, materializada en los objetivos que se relacionan con el proceso de escritura en cada grado. 
Es necesario indicar que no se debe confundir las patologías del lenguaje escrito con las deficiencias que se pueden presentar en la asimilación de la lectura y la escritura debido a la utilización de métodos inadecuados de enseñanza, o un mal manejo pedagógico. El lenguaje escrito constituye desde su inicio una actividad organizada y voluntaria, con un análisis consciente, de la composición sonora de cada verbalización.

El carácter analítico, detallado de esta actividad se conserva durante mucho tiempo y solo en las etapas tardías de formación, la escritura se puede transformar en un hábito automatizado y complejo. Un aspecto importante en la precisión de la composición sonora de la palabra es la articulación correcta, la escritura de los niños, sobre todo en los primeros grados de la enseñanza primaria depende de la articulación sonora y consiste en la pronunciación de las palabras durante la escritura, lo que forma el componente motor de su análisis sonoro.

En este proceso de aprendizaje y sistematización de la escritura no todos los escolares reaccionan de igual forma ante las exigencias que para cada grado están establecidas, existiendo alumnos que llegan a poseer afectaciones en esta área. Al evaluar sus resultados se observan la existencia de problemas en la escritura que contribuyen poderosamente al fracaso escolar, o lo que es peor a la desmotivación ante el estudio con graves consecuencias en las restantes asignaturas que influyen también en la esfera afectiva de la personalidad del escolar.

Desde que el niño comienza las clases de escritura, es decir, desde los inicios de la etapa de adquisición del aprendizaje, ha ido ejercitándose gradualmente en el análisis fónico de las palabras, que constituye la base de su representación gráfica.

Todos los escolares deben escribir legiblemente, para que lo escrito pueda leerse y para que, a la vez ellos sean capaces de leer los manuscritos de otros y entender su propia escritura. El desarrollo y funcionamiento óptimo del lenguaje escrito depende de la evolución y situación de varios factores, el lenguaje oral, la gnosis óptico-espacial, la motricidad fina, manual y espacial, la función reguladora y planificadora del córtex frontal, el sistema de enseñanza establecido, estos factores se presentan con deficiencia aisladamente o en conjunto, se dificulta el desarrollo del lenguaje escrito con un carácter más o menos transitorio o estable.

Gracias al dominio de la lengua escrita se realiza el proceso de apropiación de la cultura, conocimientos y técnicas de la sociedad en que vivimos. De ahí que un cabal conocimiento de los diferentes tipos de dificultades, sus características y manifestaciones, su evaluación, así como el adecuado enfoque de la prevención e intervención sean de suma importancia para quienes desarrollamos una actividad profesional con los escolares que presentan estas dificultades.

Los estados patológicos del lenguaje escrito reciben el nombre de agrafia y alexia en los casos más severos. Lo que significa etimológicamente sin (a) escritura (grafía) lectura (lexía) respectivamente. Cuando son menos generalizados se denominan disgrafía y dislexia. La partícula dis indica que su afectación es parcial.

Algunos investigadores al referirse a las causas que originan dificultades en el lenguaje escrito refieren: Pérez (2013) que la disgrafía es un trastorno específico y parcial del proceso de escritura, donde se manifiestan insuficiencias para asimilar y utilizar los símbolos gráficos del lenguaje, reflejado en los procesos de identificación, reproducción e interpretación. Al respecto, Pérez (1985) hace un análisis más detallado al referirse a sus características y causas, enmarcando estas en trastornos de lateralización, de deficiencias psicomotoras, del esquema corporal y de las funciones perceptivas motrices.

A partir de hacer un análisis de los conceptos dados por investigadores del tema la autora asume el concepto dado por Duardo y Llopiz (2018), la cual se afilia al brindado por Santos (2001) quien precisa que la disgrafía es un "trastorno de la forma del trazado de los 
signos gráficos de carácter perceptivo motriz que afecta la calidad de la escritura y es, generalmente, de etiología funcional". Teniendo en cuenta los criterios existentes sobre la clasificación de las disgrafías en el proceso de escritura la autora se afilia a la realizada por Quiroz (1989) el cual las clasifica en óptica, acústica y motriz o caligráfica.

- Disgrafía óptica: Se fundamenta en la alteración de la percepción y representación visual. Se observan dificultades para reconocer letras aisladas y no las relacionan en los sonidos correspondientes. En estos casos, una misma letra puede percibirse de forma diferente en uno u otro momento, lo que conduce a cambios sistemáticos de grafemas semejante desde el punto de vista gráfico como, por ejemplo: $\mathrm{u}-\mathrm{v} ; \mathrm{d}-\mathrm{g} ; \mathrm{n}-\mathrm{m}$.

- Disgrafía acústica: Se afecta la percepción auditiva del lenguaje manifestándose en el insuficiente desarrollo de la diferenciación acústica de los fonemas y el análisis y síntesis de la composición sonora de las palabras.

Se presentan dificultades en el reconocimiento de fonemas semejantes por sus características acústico - articulatorias $(\mathrm{p}-\mathrm{b}, \mathrm{t}-\mathrm{d}$, ch - $1 \mathrm{l})$ y en la estructura se confunden, se combinan.

Para el desarrollo de esta investigación se hizo necesario profundizar con más énfasis en la disgrafía caligráfica, pues en este tipo de disgrafía se afecta la calidad de la escritura y en concreto el grafismo en sus aspectos grafomotores. Las dificultades pueden ser de tres tipos:

- Referente a los alógrafos.

- $\quad$ Referente a los patrones motores.

- $\quad$ Referente a la organización general.

La escritura referente a los alógrafos se caracteriza por la confusión de alógrafos, la mezcla de letras mayúsculas y minúsculas, la mezcla de diferentes escrituras, la adición u omisión de rasgos, etc. En la referente a los patrones motores, los niños presentan letras mal dibujadas, letras excesivamente grandes o excesivamente pequeñas, letras mal inclinadas y rasgos de las letras desproporcionadas. En la escritura referente a la organización general, aparecen líneas inclinadas, grandes espacios entre las líneas, palabras amontonadas y márgenes demasiado amplios.

En los trabajos escritos se observa la pérdida o desviación del renglón y desfiguración de grafías. En algunos casos, se dificulta la coordinación para reproducir los movimientos articulatorios por alteración en la cinestesia articulatoria lo que se refleja directamente en la escritura en la que observan con frecuencia omisiones de letras y sílabas, particularmente de vocales, así como de consonantes cuyos fonemas tienen semejanza.

Por lo antes planteado resulta de considerable importancia el conocimiento de las características de la disgrafía caligráfica tales como:

- Dificultades en la definición de la lateralidad manual para escribir.

- Dificultad en la regulación de la fuerza de la mano al escribir (uso de mucha o muy poca fuerza).

- Dificultad para escribir recto y en paralelo en el papel no rayado.

- Pobre continuidad del movimiento en la escritura.

- Escritura muy lenta.

- Inversión o rotación de símbolos escritos (particularmente letras y dígitos).

- Dificultad para mantener la secuencia correcta de las letras, en una palabra.

- Escritura ilegible.

- Dificultad en el copiado de símbolos, palabras y textos.

- Dificultad en el dictado de palabras o textos. 
- Dificultad para escribir textos con coherencia semántica y/o sintáctica.

- Dificultad en la redacción de textos (falta de ajuste al tema, de orden lógico, de calidad en las ideas).

Existen además otros criterios de clasificación relacionadas con las disgrafías pues algunos autores definen las siguientes: la disgrafía acústico - articulatoria, la disgrafía acústica y la disgrafía agramática, mientras que otros autores de habla hispana como Fernández (2001) y Giordano (2004) clasifican las disgrafías como: disgrafía disléxica y disgrafía caligráfica. La disgrafía surge a consecuencia de diversas causas las cuales pueden considerarse como factores personales predisponentes o como factores condicionantes. Entre los factores predisponentes encontramos las de tipo biológica, tales como: los factores hereditarios, disfunciones no demostrables en el sistema nervioso central, inmadurez en determinadas áreas de la corteza cerebral, retrasos en la coordinación inter hemisférica y retraso en la integración intersensorial. También se encuentran las psicológicas, tales como: inmadurez de los procesos psicológicos básicos y en el desarrollo de la organización espacio - temporal y la dominancia lateral, funcionamiento estratégico insuficiente, estilos cognitivos excepcionales, falta de interés por el estudio. Los que actúan como factores condicionantes dentro del contexto familiar son: la pobre estimulación de procesos psicológicos básicos e inadecuada motivación por el aprendizaje. En el contexto escolar está la insuficiente preparación previa para el aprendizaje, situaciones conflictivas y estresantes alrededor del proceso de aprendizaje, empleo de métodos, procedimientos y recursos rígidos y el descuido de las particularidades individuales de los escolares.

Existe la tendencia a agrupar las causas en función del diagnóstico multilateral e integral, en estos casos se destacan las siguientes:

- Las madurativas donde se incluyen en este grupo las dificultades neuropsicologías, la mala lateralización, el insuficiente desarrollo psicomotor, del esquema corporal, de las capacidades perceptivo - motrices, el insuficiente desarrollo del oído verbal, de la percepción fonemática y los retardos en el desarrollo del lenguaje oral.

- Las pedagógicas donde se constata una enseñanza inflexible, rígida, aplicada a todos los alumnos por igual sin tener en cuenta las particularidades individuales, las vías de acceso para el aprendizaje, el diseño de objetivos ambiciosos, sin considerarlas potencialidades de los alumnos, así como la selección de los métodos, medios y evaluación de la enseñanza en relación con las particularidades de los sujetos que aprenden.

- -Las motivacionales donde los psicopedagogos plantean que la insuficiente preparación y motivación familiar por la adaptación del escolar al proceso escolar puede provocar inadaptación escolar.

Es cierto que los métodos educativos incorrectos y la desorganización en el régimen de vida del escolar puede obstaculizar el desarrollo de la personalidad y madurativo general, lo cual repercute negativamente en el aprendizaje como proceso organizado y consciente al que se enfrentan los alumnos.

Las investigaciones realizadas por diferentes autores muestran que las diferentes formas de disgrafía se presentan por lo general combinadas, debido a que se observa en los sujetos con estos trastornos, manifestaciones diversas que evidencian debilidad en las conexiones internalizadoras y por tanto en la fijación de los patrones sonoros, visuales y motrices de los procesos de lecto - escritura.

Sobre este tema la autora considera que se hace necesario preparar al docente de la Enseñanza Especial no solo para corregir la disgrafía motriz del escolar, sino también para favorecer un adecuado desarrollo de la comunicación en el entorno escolar, pues debe conocer o estar preparado para detectar los trastornos de la lectoescritura, por eso es necesario que 
conozca los pasos dados por Marga Ojeda Martínez y a los cuales se acoge la autora de esta investigación por considerarlos muy importantes, los mismos son:

- estudio del esquema corporal.

- exploración de las nociones temporo- espaciales, de la lateralidad.

- percepción fonemática (análisis y síntesis de la composición sonora de la palabra)

- discriminar un sonido en sílabas, palabras.

- seleccionar tarjetas ilustradas donde esté el sonido.

- pensar palabras con determinado sonido.

- repetir sílabas con fonemas correlativos.

Es la enseñanza de la lengua materna la que tiene como objetivo fundamental dotar a los educandos del instrumento que les permita comunicarse amplia y acertadamente en el ámbito social, tanto en forma oral como escrita, demostrando el carácter instrumental de esta materia para el cumplimiento de importantes funciones de la lengua tales como: proporcionar el intercambio humano, organización, desarrollo y expresión del pensamiento y la regulación de la conducta.

Por lo que el trabajo del maestro consiste en prevenir, corregir y/o compensar los trastornos del lenguaje y comunicación, para el desempeño de sus funciones requiere también del apoyo de otros especialistas para solucionar los problemas del diagnóstico de sus escolares y poder desarrollar una clase efectiva, en ellos debe estimular el desarrollo del lenguaje y corregir los trastornos de la comunicación escrita, evaluar, rehabilitar, educar, organizar, controlar y dirigir, estas actividades de conjunto con la logopeda y la familia.

Esta labor va dirigida a la corrección, desarrollo y enriquecimiento del lenguaje y las habilidades comunicativas que constituyen un fundamento decisivo para el proceso de enseñanza aprendizaje a partir de los objetivos y contenidos de cada grado.

Por consiguiente, se hace necesario que los docentes tengan dominio de los errores que puedan presentar los educandos como es el caso de las disgrafías escolares. Tal es la importancia que se le concede a la prevención y corrección de las disgrafías escolares, que varios han sido los trabajos realizados a nivel mundial sobre el tema por pedagogos, logopedas e investigadores.

En particular en Cuba esta problemática constituye una prioridad en el ámbito de la educación y se han desarrollado variadas investigaciones en este campo, entre los que se destacan: Figueredo y Aldana (1986), Rivas (2000), Mendoza y Betancourt (2004), Pérez (2005), Navarro y Quintero (2006), los cuales han coincidido en que para realizar un efectivo tratamiento de la disgrafía en la etapa escolar el maestro debe profundizar en los siguientes aspectos:

- El estudio anamnésico: para precisar el nivel de evolución de las funciones físicas y psíquicas de la personalidad, su conducta y entorno afectivo, así como el de sus familiares.

- Estudio sociopedagógico: donde se debe tener en cuenta el nivel sociocultural en que se desarrolla el niño, así como las relaciones familiares, comienzo de la escolaridad, adaptación escolar y definición de la mano para escribir.

- Datos sobre el lenguaje: para precisar el estado de la pronunciación, función auditiva, procesos fonemáticos, vocabulario y estructuración gramatical, lectura, escritura, estado del intelecto, de las funciones perceptivo-motrices, imagen corporal, lateralidad, función visual (biológico, análisis y síntesis visual), motricidad, madurez pedagógica.

Para resolver los problemas del lenguaje escrito la Jefa de Ciclo debe ofrecer talleres al 
docente que le permitan incorporar actividades dentro de sus clases encaminadas a corregir las dificultades en la escritura que presentan los escolares. El material que se instrumentó está elaborado teniendo en consideración que la psiquis tiene un carácter activo en la regulación de la actuación y está determinada histórica y socialmente en su origen y desarrollo, en la medida que se forma y desarrolla en el proceso de la actividad y comunicación que el sujeto establece en el medio socio histórico en que se desenvuelve, por lo que se podrán apreciar talleres donde el alumno tiene que comunicarse con los demás e interactuar con estos en el contexto donde se desarrolla, dígase el hogar, la escuela y la comunidad, estos talleres se enriquecen por el contenido, método, medios de enseñanza que se emplean en correspondencia con el objetivo propuesto y su relación con los objetivos y contenidos de la asignatura del currículum escolar así como las carencias y demanda de los escolares para lograr un aprendizaje al propiciar la compensación de los trastornos que se manifiestan en este proceso.

Los autores proponen organizar los talleres por la influencia positiva de estos en la corrección y compensación de las dificultades que se presentan al escribir y lograr efectividad en el proceso de enseñanza aprendizaje de los escolares. Las exigencias actuales del trabajo preventivo se pueden interpretar desde una nueva dimensión del trabajo correctivo compensatorio, lo que exige una clara comprensión de los postulados de Vygotsky, L.S. por ejemplo, cuando él decía: la ley de la compensación se aplica de la misma manera al desarrollo normal y al complicado. Vigotsy (1985).

Por ello hoy en nuestro país se les presta especial atención a los niños con necesidades educativas especiales que puedan presentar desde edades tempranas trastornos en la lectura y la escritura haciendo énfasis principalmente en los casos de disgrafía debido a la importancia que representa corregir estas deficiencias; en la actualidad nuestro sistema nacional de educación ha depositado en nuestros educadores la más plena confianza y todos los medios necesarios para dar solución a esta problemática, confiando en ellos la tarea más hermosa que existe, educar a las nuevas generaciones y en especial a los escolares que presentan discapacidad intelectual.

Bokova (2014), plantea que: Un estudio en Latinoamérica sobre analfabetismo funcional, concluyó que cuatro años de escolaridad no bastan para adquirir las competencias pertinentes en lectura y escritura; se requieren 6 o 7 años de escolaridad y que es primordial invertir en la primera infancia con los mejores docentes porque una mala calidad educativa influye en el grado de alfabetismo de los futuros jóvenes y adultos.

El proceso de atención a personas con disgrafía, como todo tipo de actividad de aprendizaje, tiene bases organizativas generales que se ajustan y no se contraponen a las metodologías específicas preestablecidas para cada contenido del programa, sin embargo, el análisis de la instrumentación de este proceso en la práctica induce a señalar que existen deficiencias en los docentes para la realización de acciones teniendo en cuenta las potencialidades que estas personas poseen, para lograr un verdadero proceso de atención a la diversidad.

El análisis de los planes de preparación en diferentes cursos escolares y la experiencia práctica en la preparación de los docentes evidenció que esta no satisface la preparación de los mismos para la corrección de la disgrafía en los escolares con discapacidad intelectual de la Escuela Especial.

El desarrollo en estos escolares se caracteriza por presentar dificultades para conocer el mundo que le rodea y por tanto muestran carencias, falta de progresos y demora significativa en alcanzar los logros del desarrollo en el lenguaje.

Poseen potencialidades del desarrollo; este aspecto resulta de gran importancia en el proceso de la enseñanza de la lectoescritura, pues estos niños muchas veces son tan capaces 
como los otros niños de su edad. Sus posibilidades de desarrollo están relacionadas con las oportunidades que se les ofrece tanto en la familia como en la comunidad.

En la actualidad se labora en el perfeccionamiento continuo del currículo, desde una perspectiva de flexibilidad, funcionalidad, contextualización y protagonismo de las instituciones, la familia y la comunidad. La escuela para alumnos con discapacidad intelectual trabaja con el objetivo de lograr el desarrollo máximo de las potencialidades.

El lenguaje de los escolares con discapacidad intelectual presenta regularidades que tienen una estrecha relación con los aspectos de carácter cognitivo e intelectual. En estas manifestaciones no sólo influye la relación pensamiento-lenguaje, también hay algunas malformaciones anatómicas y determinadas alteraciones perceptivas y motrices.

La aparición retardada del lenguaje y la lentitud en su desarrollo limitan la relación de los niños con discapacidad intelectual con el medio que les rodea, lo que incide negativamente en el desarrollo de sus motivaciones e intereses cognoscitivos.

El lenguaje se caracteriza por la aparición tardía de todas sus formas, apropiación insuficiente del aspecto semántico del lenguaje materno, desarrollo insuficiente de la pronunciación y del oído fonemático, limitaciones en la comprensión del lenguaje ajeno, uso estereotipado de las formas gramaticales y limitadas iniciativas verbales espontáneas.

Adquieren los mismos fonemas, aunque tardíamente y con dificultades en la articulación de algunos de ellos. Todo ello influye en la aparición de las disgrafías.

El desarrollo del lenguaje suele retrasarse sobre todo en aquellos que presentan un grado moderado, severo y profundo de discapacidad intelectual. Con mayor o menor fluidez y dominio del habla las personas con discapacidad intelectual logran comunicarse, ya sea de forma verbal o extraverbal.

Hay niños que emiten frases cortas en las que no siempre se conserva una estructura gramatical adecuada, mientras que en otros casos emplean palabras aisladas, en otros hay ausencia total del habla, con pocas iniciativas para establecer diálogos y conversaciones. Sus habilidades lingüísticas pueden variar desde la ausencia total del habla, el uso de palabras aisladas, empleo de frases cortas sin una coordinación adecuada, emisión de frases y oraciones simples con un pobre vocabulario; también se encuentran los que se expresan con bastante corrección, según la estimulación recibida en los diferentes contextos de convivencia.

Existen diferencias importantes entre el vocabulario pasivo y activo, su pobreza está condicionada por las limitaciones en la actividad cognoscitiva y por el papel peculiar que desempeña el lenguaje en la transformación de la psiquis de estos niños.

Suelen emplear en su lenguaje un número reducido de construcciones gramaticales y pueden desarrollar habilidades lectoras a un nivel aceptable, aunque la fluidez, corrección, expresividad y comprensión se encuentran afectadas. El enriquecimiento de la actividad cognoscitiva y la estimulación del desarrollo de sus intereses cognitivos desde las primeras edades contribuyen a enriquecer el vocabulario y la construcción gramatical en su expresión oral y escrita.

El grado o nivel de dificultades que puede presentar el niño en el proceso de la escritura, depende del tamaño y la intensidad que tenga la lesión, daño o afectación del sistema nervioso central; también depende de la calidad del medio social y ambiental donde éste se desarrolla, así como de la aplicación oportuna de las estrategias de estimulación y de las acciones educativas por parte de la familia y los especialistas.

La escritura se puede ver afectada en gran o menor medida debido también a las 
insuficiencias que presentan en relación con la orientación espacio-temporal, es poco el desarrollo de habilidades para orientarse de acuerdo con las direcciones cambiantes (a la derecha, a la izquierda, hacia arriba, hacia abajo, hacia delante, hacia atrás), lo que influye en la comprensión de las relaciones espaciales.

La diferenciación de las mitades derecha e izquierda del cuerpo resulta difícil para los niños con discapacidad intelectual de los primeros grados, siendo esto una condición previa para la distinción de las direcciones derecha e izquierda en el espacio circundante. Esto puede dar origen a las disgrafías ópticas y motrices.

Las áreas del desarrollo de la personalidad seleccionadas ofrecen la posibilidad de una aproximación entre los objetivos de la educación general y la especial, a lo que se incorpora lo distintivo de la especialidad tanto en la declaración del área para el desarrollo de las habilidades laborales como en la integración de dimensiones importantes del lenguaje oral y escrito, en este componente se trabaja:

- La asimilación de la lengua materna y la ejercitación para la pronunciación.

- Ampliación de la comprensión del lenguaje.

- Ampliación del vocabulario.

- Uso apropiado de relaciones verbales (lugar).

- Uso de tiempos verbales.

- Asimilación de patrones sensoriales.

- Acciones de identificación, comparación y modelación.

- Acciones de pensamiento.

- Pensamiento en acción y en imágenes.

- Atención, memoria e imaginación.

- Apropiación del sistema de nociones, rasgos básicos de conceptos y habilidades intelectuales.

- Dominio de procedimientos y estrategias de aprendizaje básicas de español y matemáticas.

- Ejecución de órdenes sencillas.

- Dominio de la lengua u otras alternativas de comunicación.

- Uso correcto de textos y otros materiales docentes con el empleo del lenguaje facilitado.

En cuanto al desarrollo de la expresión oral:

- Conversar exponiendo las ideas con un orden lógico a través de dramatizaciones y representaciones de cuentos.

- Memorizar y escuchar poesías, adivinanzas y trabalenguas que favorezcan la corrección de los problemas en el lenguaje.

- Leer con corrección utilizando la síntesis oracional teniendo en cuenta:

- Pausas.

- Pronunciación.

- Entonación.

- Comprender los textos de las lecturas, narraciones, descripciones a través de:

- El conocimiento del significado de nuevas palabras, expresiones y refranes.

- Relacionar el texto, ilustraciones.

- Reconocer las ideas más importantes expresadas en el texto.

- Ordenar cronológicamente sucesos.

- Identificar el mensaje o moraleja.

- Identificar las acciones a partir de la descripción oral.

- En cuanto al desarrollo de la expresión escrita:

- Escribir nombres de personas, animales y cosas. 
- Ampliar oraciones dadas.

- Redactar oraciones relacionadas entre sí, como condición indispensable para el posterior dominio de la redacción de párrafos.

- Escribir los artículos, él, la, les, los, delante de los sustantivos.

- Ordenar, formar, escribir, redactar y distinguir oraciones enunciativas, exclamativas e interrogativas.

- En cuanto al desarrollo de habilidades ortográficas.

- Usar la mayúscula al inicio de las oraciones y en los sustantivos propios.

- Utilizar los signos de puntuación estudiados: punto final, signos de interrogación, exclamación, el guión y la diéresis.

- Distinguir las sílabas tónicas de las palabras.

- Memorizar y escribir el alfabeto, logrando un nivel de familiarización con el diccionario.

- Copiar, transcribir y escribir al dictado palabras y oraciones con los grafemas estudiados.

- Identificar y usar correctamente reglas ortográficas como m antes de p y b y el uso de la b, así como otras que resulten necesarias para una correcta escritura.

La formación permanente o continua del profesorado es una demanda educativa ampliamente solicitada, dentro de la compleja serie de actividades personales y profesionales que componen el desarrollo docente. Dado que no todo debe ser enseñado y aprendido al principio, aunque el profesorado tenga experiencia, el desarrollo profesional es una parte esencial de la preparación de todo maestro.

\section{Conclusiones}

1-El análisis de los fundamentos teóricos, permitió determinar lo referido a la corrección de la disgrafía caligráfica, así como la atención a estas disgrafías y las características del lenguaje en los escolares con discapacidad intelectual. En este caso se consideró oportuno el empleo de las áreas del desarrollo de la personalidad, por supuesto con su correspondiente adecuación a las características de los escolares objeto de estudio.

2-La determinación del diagnóstico de las potencialidades y necesidades de los escolares con discapacidad intelectual de la Escuela Especial "Osvaldo Cabrera Alonso" del municipio de Palmira favorecerá el desarrollo de la elaboración de la propuesta del sistema de talleres.

\section{Referencias}

Anderson G. (2014). Las etapas del grafismo.

Akudovich, S. (2004). Estudio de la ZDP de los alumnos con retraso mental como parte de la atención a la diversidad. La Habana: CELAEE.

Asociación Americana de Psiquiatría. (2013). Guía de consulta de los criterios diagnóstico del DSM-5. Washington, DC, London, England.

Abad, M., Rivero N., \& Morales, Á. (2018). La preparación de los docentes para la prevención y corrección de los errores de segregación y condensación. Revista Atlante: Cuadernos de Educación y Desarrollo.

Addine, F. (2004). Didáctica, teoría y práctica. La Habana: Pueblo y Educación.

Agenda 2030. (2020). La Habana: Pueblo y Educación.

Árias, B. G et. al. (1999). Educación, desarrollo, evaluación y diagnóstico desde el enfoque histórico cultural. La Habana: Universidad de La Habana.

Árias, B. G. (2002). Retraso Mental. Madrid Alianza.

Ayala, R. Y. (2012). Conjunto de actividades para corregir y/o compensar las fallas o síntomas de la Discalculia escolar en escolares con trastornos de la comunicación. La Habana. Recuperado de: https://www.unesco.org 
Betancourt. J. (2003). La comunicación educativa en la atención a niños con necesidades educativas especiales. La Habana: Pueblo y Educación. Recuperado de: https://www.cubaeduca.cu

Bokova, I. (2014). Obtenido de La formación docente es clave para la calidad educativa. Recuperado de: http://www.revista-critica.com

Compendio de pedagogía (2002). La Habana: Pueblo y Educación.

Delgado, G. O. Díaz, E. (2016). Caracterización de disgrafía en niños y niñas. Rev de información Científicam 95(6), 883-892. Recuperadeo de; http://www.revinfcientifica.sld.cu/index.php/ric/article/view/42/1516

Duardo, E. (2018). Orientación a la familia para el apoyo a la correción de la disgrafía motriz en un escolar de la enseñanza primaria. Santa Clara.

Fernández, G. (2013). Logopedia 2da Parte. La Habana: Pueblo y Educación.

Hernández, R. (2014). Metodología de la investigación 6ta edición. La Habana: Pueblo y Educación.

Hernández, M. (2017). La corrección de la disgrafía disléxica en un escolar con diagnóstico en el desarrollo psíquico. Santa Clara.

Loaiza, Z. Tamayo, A. Zona, R. (2015). El pensamiento crítico en la educación. Rev. latinoamericana de estudios educativos, 11(2).

López, J. (2009). Propuesta de capacitación a los docentes de segundo grado de la Escuela Corina Rodríguez Morera para la corrección de las disgrafias pedagógicas. La Habana: Ministerio de Educación.

López, R. (1980). Glosario de términos logopédicos. La Habana: Pueblo y Educación.

Love, R. Webb, G. (2006). Neurología para los especialistas del habla y del lenguaje. La Habana: Ciencias Médicas.

Luria, A. (1977). Las funciones corticales superiores del hombre. La Habana: CientíficoTécnica.

Lubovski, I. (1976). Particularidades de la actividad nerviosa superior de los escolares con retraso mental. La Habana: Pueblo y Educación.

Medina, I., Rivero, N. \& Morales, A. (2013). ¿Es Posible prevenir y corregir los errores de segregación y condensación? Revista Conrado, 9(41).

Miranda, R.L. (2008). Propuesta de actividades encaminadas a preparar a los niños bajos en el diagnóstico de preescolar para la adquisición de la lecto-escritura en 1er grado. Cuba: ISP Conrado Benítez García.

Morales, M.E. (2004). Intervención logopédica en los alumnos con Retraso mental. La Habana: Pueblo y Educación.

Padrón, I. (2002). Logopedia 2da parte. La Habana: Pueblo y Educación.

Quiroz, J. (1980). El lenguaje lectoescrito y sus problemas.

Scrich, A., Cruz, L. Bembibre, D. Torres, I. (2017). La dislexia, la disgrafia y la discalculia: sus consecuencias en la educación ecuatoriana. Revista Archivos Meducos de Camagüey, 21(1).

Solano, G. K. (2015). La disgrafía estudiantil en el proceso de enseñanza y aprendizaje y la formación profesional del maestro. Universidad técnica de Machala.

Vygotsky, L.S. (1985). Obras completas: T.V. La Habana: Pueblo y Educación. 